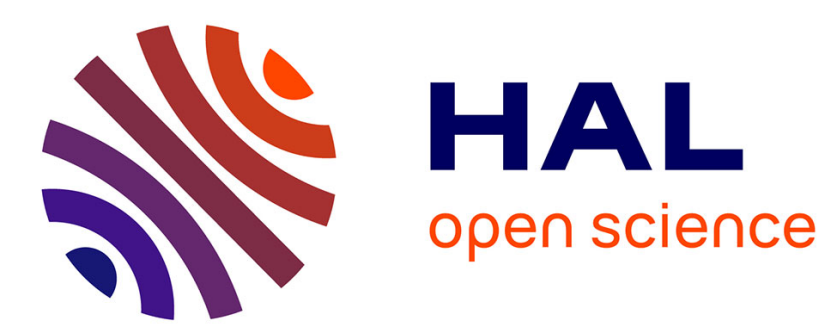

\title{
Site stability and pipe diffusion of hydrogen under localised shear in aluminium
}

Yu Wang, D. Connetable, Döme Tanguy

\section{To cite this version:}

Yu Wang, D. Connetable, Döme Tanguy. Site stability and pipe diffusion of hydrogen under localised shear in aluminium. Philosophical Magazine, 2019, 99 (10), pp.1184-1205. 10.1080/14786435.2019.1576935 . hal-02285882

\section{HAL Id: hal-02285882 \\ https://univ-lyon1.hal.science/hal-02285882}

Submitted on 18 Nov 2019

HAL is a multi-disciplinary open access archive for the deposit and dissemination of scientific research documents, whether they are published or not. The documents may come from teaching and research institutions in France or abroad, or from public or private research centers.
L'archive ouverte pluridisciplinaire $\mathbf{H A L}$, est destinée au dépôt et à la diffusion de documents scientifiques de niveau recherche, publiés ou non, émanant des établissements d'enseignement et de recherche français ou étrangers, des laboratoires publics ou privés. 


\section{OATAO \\ Open Archive Toulouse Archive Ouverte}

\section{Open Archive Toulouse Archive Ouverte (OATAO)}

OATAO is an open access repository that collects the work of Toulouse researchers and makes it freely available over the web where possible

This is an author's version published in: http://oatao.univ-toulouse.fr/25104

Official URL: https://doi.org/10.1080/14786435.2019.1576935

\section{To cite this version:}

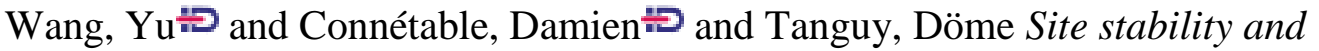
pipe diffusion of hydrogen under localised shear in aluminium. (2019)

Philosophical Magazine, 99 (10). 1184-1205. ISSN 1478-6435

Any correspondence concerning this service should be sent to the repository administrator: tech-oatao@listes-diff.inp-toulouse.fr 


\title{
Site stability and pipe diffusion of hydrogen under localised shear in aluminium
}

\author{
Y. Wang ${ }^{\mathrm{a}, \mathrm{b}}$, D. Connétable ${ }^{\mathrm{b}}$ and D. Tanguy ${ }^{\mathrm{a}}$
}

${ }^{a}$ Institut Lumière Matière, Univ Lyon, Université Claude Bernard Lyon 1, CNRS, Villeurbanne Cedex, France; ${ }^{b}$ CIRIMAT UMR 5085, CNRS-INPT-UPS, École Nationale d'Ingénieurs en Arts Chimiques et Technologiques (ENSIACET) 4, Toulouse Cedex 4, France

\begin{abstract}
This paper studies the effect of a plastic shear on the tetrahedral vs. octahedral site stability for hydrogen, in aluminium. Based on Density Functional Theory calculations, it is shown that the tetrahedral site remains the most stable site. It transforms into the octahedral site of the local hexagonal compact structure of the intrinsic stacking fault. The imperfect stacking is slightly attractive with respect to a regular lattice site. It is also shown that the shearing process involves a significant decrease of the energetic barrier for hydrogen jumps, at half the value of the Shockley partial Burgers vector, but not in the intrinsic stacking fault. These jumps involve a displacement component perpendicular to the shearing direction which favours an enhancement of hydrogen diffusion along edge dislocation cores (pipe diffusion). The magnitude of the boost in the jump rate in the direction of the dislocation line, according to Transition State Theory and taking into account the zero point energy correction, is of the order of a factor 50, at room temperature. First Passage Time Analysis is used to evaluate the effect on diffusion which is significant, by only at the nanoscale. Indeed, the common dislocation densities are too small for these effects (trapping, or pipe diffusion) to have a signature at the macroscopic level. The observed drop of the effective diffusion coefficient could therefore be attributed to the production of debris during plastic straining, as proposed in the literature.
\end{abstract}

\section{KEYWORDS}

Hydrogen; plasticity;

diffusion; ab initio simulation

\section{Introduction}

An important area of research, within the field of environmental damage of structural metallic alloys, is hydrogen embrittlement. This type of damage is intimately related to the tendency of metallic systems to form a refined dislocation structure, ahead of crack tips [1,2], among which a crack can propagate at low mechanical loads. Hydrogen is not only involved in the crack advance itself 
but is also responsible for the buildup of the specific dislocation structure. Some related elementary plastic mechanisms (for a review see [3]), such as an enhanced localisation in slip bands, have been recently quantified [4,5]. Also of importance is the modelling of hydrogen diffusion in such a microstructure [6], i.e. to include the presence of dislocations in continuum diffusion. This has essentially been viewed as a stress bias, with an additional trapping at the core of the dislocation [7-9] or at debris produced by plastic strain. Pipe diffusion is not considered and only a limited amount of studies has been devoted to the subject [10]. There is still a need to investigate the details of the diffusion paths provided by the region of imperfect stacking at the core of dislocations and along stacking fault ribbons, in a similar way to what was done in the intergranular case [11].

Crack tip plasticity can also be influenced by adsorbed hydrogen $[12,13]$. This issue has recently received a special attention with the use of Density Functional Theory (DFT) calculations at crack tips $[14,15]$. The calculations show that $\mathrm{H}$ increases the stress intensity factor for dislocation emission at the tip $\left(k_{I e}\right)$, with a high influence of the adsorption site [14]. The effect of sub-surface hydrogen on dislocation emission, or of bulk hydrogen on the dissociation of the dislocation core, can be, as a first approach, modelled by calculating the generalised stacking fault energy (GSF) [16-18]. The GSF is obtained by block shearing the crystal in the direction of the Burgers vector, i.e. the bond shearing is localised in between two adjacent crystallographic planes. Two previous attempts, using DFT to evaluate the GSF, have led to fairly different results $[17,19]$, depending on the way the system was initially relaxed and the hydrogen concentration along the slip plane. A crossover of the stability between the sheared tetrahedral site and the sheared octahedral site suggests that $\mathrm{H}$ could change site during the shearing process and that it would involve a very significant reduction of the GSFs [17]. On the contrary, it was found that at high $\mathrm{H}$ concentrations, the stacking would reconstruct and that it would prevent sliding [19]. An additional motivation for the present study, that will be detailed in a forthcoming paper [20], is the evaluation of the effect of sub-surface hydrogen on, in plane, Shockley partial emission at a crack tip loaded in mixed mode I plus II [21]. In this crack-dislocation configuration, the stress intensity factor is simply related to the lowest local maximum of the GSF (the unstable stacking energy $\gamma_{u s}$ ), by the relation: $k_{I I e}\left(k_{I}\right)=\sqrt{2 \mu \gamma_{u s}\left(\Delta u_{z}\right) /(1-v)} . \Delta u_{z}$ is the elongation of the inter-planar distance normal to the slip plane, at the crack tip and $\mu$ and $v$ are elastic constants. Mode I creates an intense traction stress, perpendicular to the glide plane, which has a strong influence on the GSF. For this reason, the influence of $\Delta u_{z}$ on the site stability and diffusion barriers will also be presented.

The paper is organised as follows: first, the methods are briefly exposed; second, the site stability for a hydrogen atom in a shear plane is reported; third, the different diffusion paths and related activation energies are given for different values of shear and transverse dilatation. The impact on the stacking 
fault energy and on the effective diffusion coefficient are quantified and discussed. Finally, the main results are summarised and perspectives are given.

\section{Methods and computational setup}

The calculations consist in GSF calculations [22] where an fcc crystal is rigidly sheared along a [112] direction ( $y$-direction). Increasing the relative displacement $\Delta u_{y}$ of the upper block with respect to the lower bloc gives a first local maximum $\gamma_{u s}$, the unstable stacking energy [18], followed by the intrinsic stacking fault (ISF) (local minimum). The corresponding rigid shear is $\left(\Delta u_{y}=b=1 / 6\|112\|\right)$, where $b$ is the Burgers vector of a Shockley partial (Figure 1). As mentioned in the introduction, the GSF is sensitive to transverse stresses which can be high, for example when considering dislocation emission at crack tips [21,23]. This is taken into account by introducing a displacement $\Delta u_{z}$ normal to the glide plane (the $z$-direction is along [111]).

First, the calculations are performed with an $\mathrm{Al}-\mathrm{H}$ inter-atomic potential described in [24]. This is because the stability of the $\mathrm{H}$ atom on the various interstitial sites of the glide plane is given, to some extent, by the geometric distortion of the $\mathrm{Al}$ cage around $\mathrm{H}$ when $\Delta u_{y}$ is increased. This can be studied by a crude repulsive $\mathrm{Al}-\mathrm{H}$ interaction. The calculation being computationally fast with this scheme, the site stability and jump activation energies can be systematically evaluated for a fine grid of $\Delta u_{y}$ and two values of $\Delta u_{z}(0$ and $0.05 a_{0}, a_{0}$ being the lattice parameter of $\mathrm{Al}$ ). The Al- $\mathrm{H}$ inter-atomic potential was not specifically fitted to any data related to plasticity, but to segregation energies to surfaces, to vacancies, bulk diffusion barriers for jumps from the tetrahedral site ( $\mathrm{T}$ site) to the octahedral site (O site). So the comparison with the DFT results can also be seen as a test of the predictive capabilities of the potential.
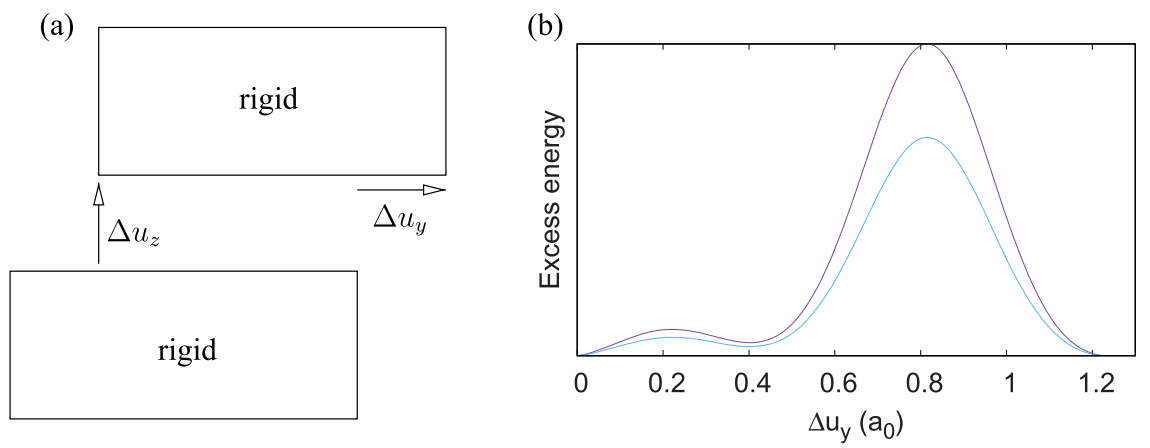

Figure 1. Schematic picture of the generalised stacking fault (GSF) calculation: (a) rigid block shearing with $\Delta u_{y}$, the shear displacement, and $\Delta u_{z}$, the displacement perpendicular to the glide plane (constant during the rigid shear). (b) Typical energy variation when a face centred cubic crystal is rigidly sheared in the [112] direction. The influence of a stress perpendicular to the glide plane is also schematically represented. 
A number of configurations are selected for DFT calculations. They are performed with the VASP (Vienna Ab-initio Simulation Package) implementation [25-27]. The Perdew-Burke-Ernzerhof (PBE) [28] exchange-correlation functional for the generalised gradient approximation (GGA) is used. A planewave basis set is employed within the framework of the projector augmented wave (PAW) [29] method. On the basis of our tests, we have determined that a cutoff energy of $400 \mathrm{eV}$ and a dense Monkhorst-Pack meshe [30] $(24 \times 24 \times 24 \mathrm{k}$ mesh for the primitive cell to sample the Brillouin zone), are adequate for a good convergence. The equilibrium theoretical lattice structure is determined by minimising the Hellmann-Feynman force on the atoms and stress on the unit cell. The convergence of force is set to $1.0 \times 10^{-3} \mathrm{e} / \AA$, respectively. Ground states properties of fcc- $\mathrm{Al}, \mathrm{H}_{2}$ and $\mathrm{Al}-\mathrm{H}$ system are thus found in excellent agreement with the literature [31].

To simulate the block shearing process, we use a slab composed of 8 atomic layers in the [111] direction. A large vacuum space of 8 atomic layers parallel to (111) plane is added between the periodically repeated slabs to avoid the interactions between two successive slabs. The top 4 atomic layers of the system are then sheared against the remaining 4 layers on the (111) plane in the [11 $\overline{2}]$ direction. For the case of pure $\mathrm{Al}$, each layer of the simulation box contains only one atom. The sliding process is rigid. Tests with 12 atomic layers were performed to examine the vacuum effect. The GSF energies show no significant differences.

Evaluating the effect of $\mathrm{H}$ on the GSF involves inserting an $\mathrm{H}$ atom in the $\mathrm{T}$ or $\mathrm{O}$ sites. On the one hand, in Rice's model, the shearing process is rigid. On the other hand, the DFT calculations in [31] show that, in bulk Al, the relaxations around the $\mathrm{H}$ atoms are very important because they invert the stability of the $\mathrm{O}$ and $\mathrm{T}$ sites. Therefore, it was chosen to perform a local relaxation where the atoms within a radius of $0.74 a_{0}$ around the $\mathrm{H}$ atom are relaxed while the others are kept rigid during the GSF calculation. $a_{0}$ is the lattice parameter. Only the $4 \mathrm{Al}$ atoms in the first neighbour position of the initial $\mathrm{T}$ position are relaxed. This number goes up to 7 during the shear process and then down to 6 in octahedral position. $0.74 a_{0}$ is a distance in between the first $\mathrm{Al}$ neighbour distance in octahedral position $\left(0.5 a_{0}\right)$ and the second neighbour position in tetrahedral position $\left(0.83 a_{0}\right)$. As a consequence, a much larger simulation box ( 16 atoms per layer) is used when the $\mathrm{H}$ impurity is considered. As discussed above, a tiny displacement $\Delta u_{z}$ is imposed perpendicular to the shearing plane. In a forthcoming paper [20], a comparison of the critical stress intensity factor, obtained from $\gamma_{u s}$ values, with this relaxation procedure, and the direct simulations of dislocation emission by Molecular Statics, confirms that the use of such relaxations is the physical way to introduce interstitial solute relaxations in the GSF calculations.

Using the GSF energy calculations in pure $\mathrm{Al}$, the hydrogen segregation energy $\Delta E_{\text {seg }}$ on the glide plane, at each shear step $\Delta u_{y}$, can be obtained by 
simply comparing the GSF energies with and without $\mathrm{H}$ :

$$
\Delta E_{s e g}\left(\Delta u_{y}, \Delta u_{z}\right)=E^{\mathrm{Al}+\mathrm{H}}\left(\Delta u_{y}, \Delta u_{z}\right)-E^{\mathrm{Al}}\left(\Delta u_{y}, \Delta u_{z}\right)-E_{0}^{\mathrm{Al}+\mathrm{H}}+E_{0}^{\mathrm{Al}}
$$

where $E^{\mathrm{Al}+\mathrm{H}}$ and $E^{\mathrm{Al}}$ are energies of deformed structures with and without $\mathrm{H}$ respectively, $E_{0}^{\mathrm{Al}+\mathrm{H}}$ and $E_{0}^{\mathrm{Al}}$ are the energies of an $\mathrm{Al}$ bulk with and without $\mathrm{H}$, respectively.

Furthermore, the nudged Elastic Band (NEB) method [32] is used to determine the minimum energy pathways (MEP) for hydrogen migration along the glide plane. In this study, a spring force-constant of $5 \mathrm{eV} / \AA$ is used between images for the NEB calculations. The convergence of the forces for each image and size of the simulation box are the same as those for the GSF energies calculations. Similar calculations (GSF and H jump barriers) are performed with the EAM potential, with the same local relaxations, but with a much larger box (30 atomic layers and 192 atoms per layer). The results are compared to the DFT ones.

\section{Site stability under plastic shear}

First, the stability of $\mathrm{H}$ in the glide plane is studied at increasing levels of shear displacement $\left(\Delta u_{y}\right)$. In the perfect fcc lattice of $\mathrm{Al}$, the most stable interstitial site is the $\mathrm{T}$ site. The $\mathrm{O}$ site is metastable and close in energy (Table 1). In a shear plane, such as the one in Figure 2,two types of $\mathrm{T}$ sites are defined according to their height relative to the shear plane. $\mathrm{T}+$ is right above a black $\mathrm{Al}$ atom and is higher in the $z$-direction ([111]) than $\mathrm{T}$ - which is right below a Grey $\mathrm{Al}$ atom. The $\mathrm{O}$ site is located at the centre of the two sheared atomic layers. We want to understand how the stable sites for $\mathrm{H}$ are modified when an intrinsic stacking fault (ISF) is gradually formed by block shearing the system up to 0.41 $\mathrm{a}_{0}$ (the module of the burgers vector $b$ ). A simple view of the Al-H bond, in the lattice, is to consider it as a pair repulsion. The tetrahedral and octahedral cages define the positions where this repulsion is symmetric. When the glide plane is sheared, the cages are deformed and the stable position for $\mathrm{H}$ is transported

Table 1. DFT computed segregation energies (eV) as a function of the displacement shift in $y$ $\Delta u_{y}$ and of the plane separation in $z \Delta u_{z}$.

\begin{tabular}{|c|c|c|c|c|c|c|}
\hline$\left(\Delta u_{y}, \Delta u_{z}\right)$ & $(0,0)$ & $(0.2 b, 0)$ & $(0.45 b, 0)$ & $(0.6 b, 0)$ & $(0.8)$ & $(1 b, 0)$ \\
\hline $\mathrm{E}_{\text {seg }}(\mathrm{T}+)$ & 0.000 & 0.022 & -0.003 & -0.002 & 0.040 & $\begin{array}{l}-0.055^{\mathrm{a}}\left(\mathrm{O}_{\mathrm{ISF}}\right) \\
-0.066^{\mathrm{b}}\left(\mathrm{O}_{\mathrm{ISF}}\right)\end{array}$ \\
\hline $\mathrm{E}_{\text {seg }}(0)$ & $\begin{array}{l}0.080^{\mathrm{a}} \\
0.09[33] \\
0.04^{\mathrm{b}}, 0.13[31]\end{array}$ & 0.086 & 0.078 & 0.080 & 0.107 & $\begin{array}{l}0.019^{\mathrm{a}}\left(\mathrm{T}-{ }_{\text {ISF }}\right) \\
0.013^{\mathrm{b}}\left(\mathrm{T}-_{\text {ISF }}\right)\end{array}$ \\
\hline$\left(\Delta u_{y}, \Delta u_{z}\right)$ & $\left(0,0.05 a_{0}\right)$ & $\left(0.2,0.05 \mathrm{a}_{0}\right)$ & $\left(0.45 b, 0.05 a_{0}\right)$ & $\left(0.6 \mathrm{~b}, 0.05 \mathrm{a}_{0}\right)$ & $\left(0.8,0.05 \mathrm{a}_{0}\right)$ & $\left(1 b, 0.05 a_{0}\right)$ \\
\hline $\begin{array}{l}\mathrm{E}_{\text {seg }}(\mathrm{T}+) \\
\mathrm{E}_{\text {seg }}(0)\end{array}$ & $\begin{array}{l}-0.049 \\
-0.012\end{array}$ & $\begin{array}{l}-0.065 \\
-0.001 \\
\end{array}$ & $\begin{array}{r}-0.048 \\
0.019 \\
\end{array}$ & $\begin{array}{r}-0.039 \\
0.018 \\
\end{array}$ & $\begin{array}{r}-0.030 \\
0.009 \\
\end{array}$ & $\begin{array}{r}-0.013 \\
0.015 \\
\end{array}$ \\
\hline
\end{tabular}

Note: The values obtained with $\Delta u_{y}=0$ or $\Delta u_{y}=1 b$ are fully relaxed, while the others are obtained with only local relaxations. $b$ is the Burgers vector of the Shockley partial.

bZPE correction included. 


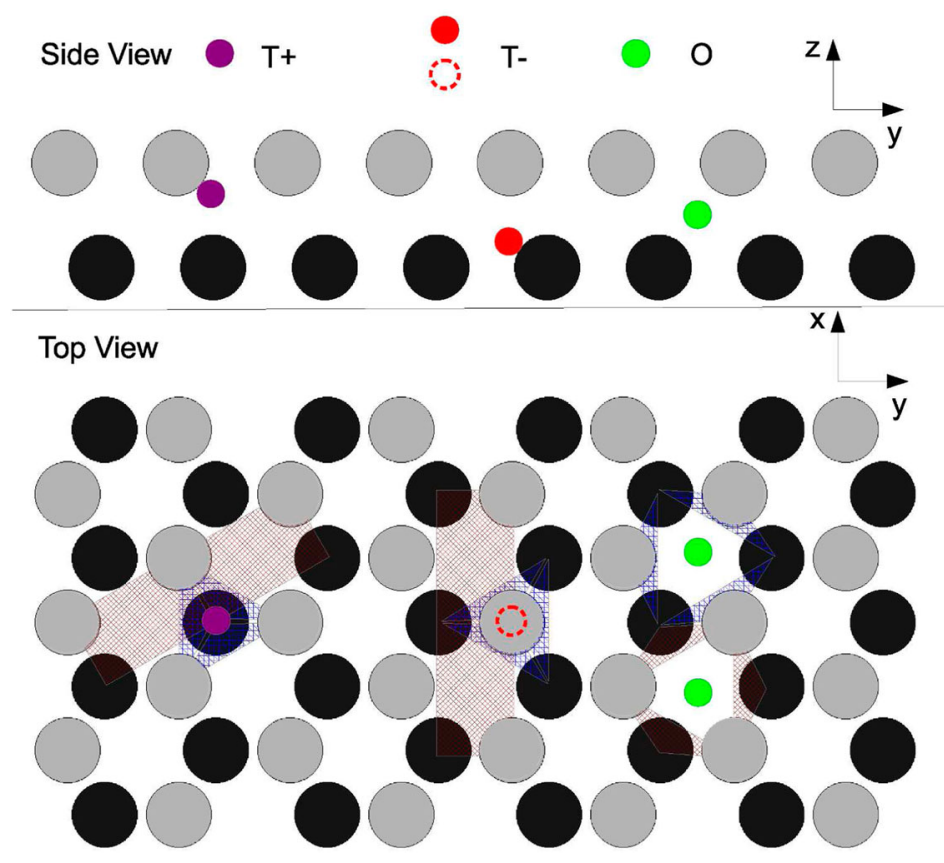

Figure 2. Side view (upper picture) and top view (lower picture) of the glide plane showing: the top Al layer (grey atoms), which can be translated to the right to form an intrinsic stacking fault, the bottom layer (black atoms) which is held fixed. The three different interstitial positions in the glide plane are $\mathrm{T}+, \mathrm{T}-$ and $\mathrm{O}$. At each value of the shear displacement $\Delta u_{\mathrm{y}}$, the first neighbours of the $\mathrm{H}$ atom are fully relaxed, while all other $\mathrm{Al}$ atoms are fixed. The $x, y$ and $z$-directions are also represented.

along the shear direction until, eventually, stability is lost and $\mathrm{H}$ moves to a new meta-stable position.

Let us consider the shearing process of Figure 2, i.e. a translation of the upper layer in the direction of positive $y$. The $\mathrm{T}+$ site is the centre of a tetrahedron composed of 3 Grey atoms (connected in Figure 2) and one black (immobile) atom. By symmetry, the $\mathrm{T}+$ site moves to the right with deformation. From a geometric point of view, the displacement shift $\Delta u_{y}=0.5 b$ can be considered as the value separating the distorted $\mathrm{T}+$ site of the fcc structure (in the $y>0$ direction) from the distorted (in the $y<0$ direction) $\mathrm{O}$ site of the ISF structure. Both EAM and DFT calculations show that the stable position for $\mathrm{H}$ smoothly moves from the distorted $\mathrm{T}+(\mathrm{fcc})$ site to the distorted $\mathrm{O}$ (hcp) site (Figure 3), as if the triangle formed by the two Grey atoms at the left of the tetrahedron and the black atom, the actual jump window for a $\mathrm{T} \rightarrow \mathrm{O}$ transition, was pushing the $\mathrm{H}$ in the $y$ direction. This mechanism does not operate in the case of the T- site (only one Grey-black bond is stretched) and the $\mathrm{H}$ atom remains in the same position during the entire shearing process (Figure 3), at least with EAM interactions (not tested in DFT). Energetically, in the case of the T+ (fcc) $\rightarrow \mathrm{O}$ (hcp) transformation, no discontinuity in the $E_{\text {seg }}\left(\Delta u_{y}\right)$ curve is seen in the vicinity of $\Delta u_{y}=0.5 b$ (Figure 4(a)), in EAM. The energy increases which implies a 


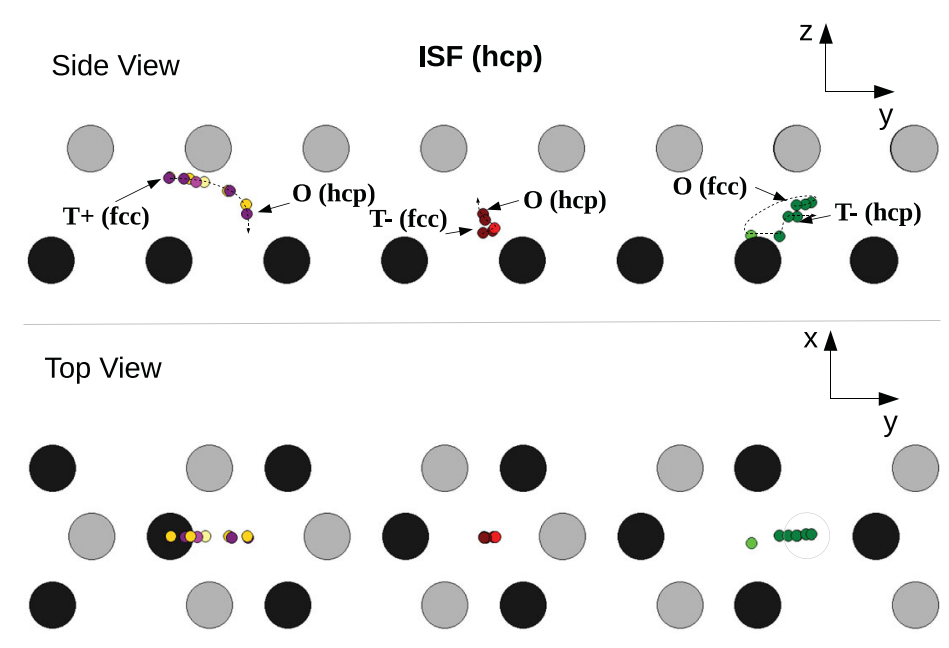

Figure 3. The Al atoms from Figure 2 are shown in their final position after the ISF was created. The small circles represent the successive $\mathrm{H}$ position during the shear, starting from the three interstitial positions of the fcc structure (Figure 2): $T+(f c c), T-(f c c)$ and $O$ (fcc). The final positions are respectively $\mathrm{O}(\mathrm{hcp}), \mathrm{O}(\mathrm{hcp})$ and $\mathrm{T}-(\mathrm{hcp})$.

small repulsion against segregation to stacking faults. The $\mathrm{O}$ (fcc) site becomes unstable, with EAM interactions, at about $\Delta u_{y}=0.4 b$. A discontinuity appears on the segregation energy versus shear curve (Figure $4(\mathrm{a})$ ). The $\mathrm{H}$ atom abruptly moves down and then gradually moves towards the distorted T- (hcp) site $\Delta u_{y}$ becomes larger than $0.5 b$ (Figure 3).

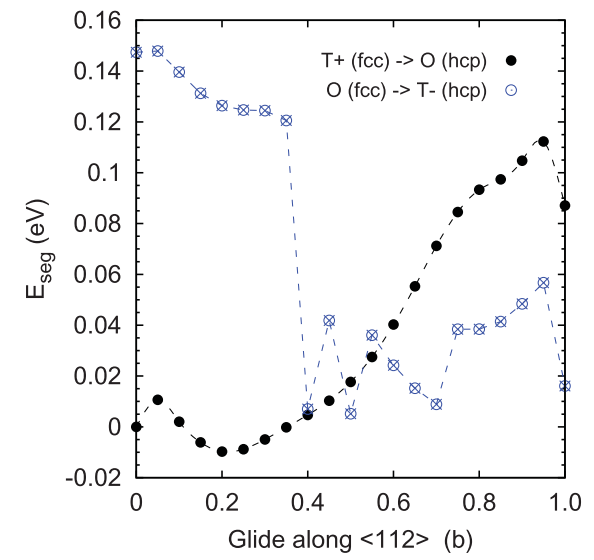

(a)

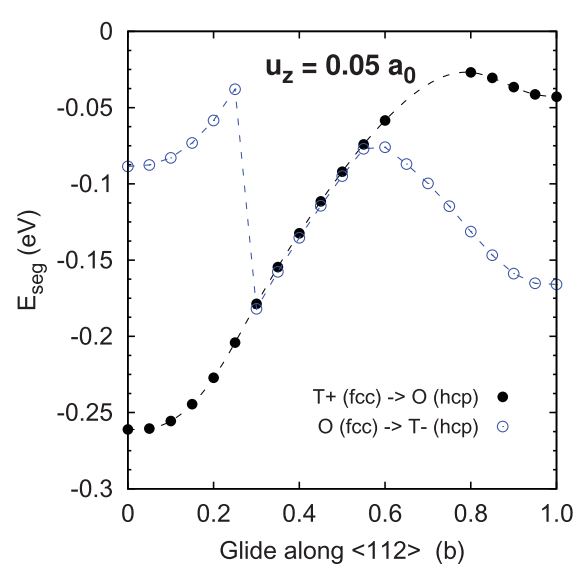

(b)

Figure 4. Segregation energies for $\mathrm{H}$ on $\mathrm{T}+(\mathrm{fcc})$ and $\mathrm{O}(\mathrm{fcc})$ sites as a function of the shear: (a) $\Delta u_{z}=0$; (b) $\Delta u_{z}=0.05 a_{0}$. Note that full relaxation is used only for the perfect fcc lattice and the ISF. Intermediate structures are minimised with the local relaxations of the neighbours of $\mathrm{H}$. This explains the discontinuities at end points of the curves in (a). Also note that all segregation energies are shifted to negative values in (b), obtained with $\Delta u_{z}=0.05 \mathrm{a}_{0}$, which reflects the tendency of $\mathrm{H}$ to segregate to open spaces (like vacancies and free surfaces, by the construction of the potential). 
In DFT, the segregation energy variation is much weaker than in EAM (Table 1). The octahedral site of the intrinsic stacking fault $\mathrm{O}_{\text {ISF }}$ is slightly attractive $(-0.066$ $\mathrm{eV}$ with full relaxation and $\mathrm{ZPE}$ correction included). The tetrahedral site $\mathrm{T}-_{\text {ISF }}$ is weakly repulsive $(+0.013 \mathrm{eV})$. Our DFT calculations do not show any crossover of the two curves $\mathrm{E}_{\text {seg }}\left(\mathrm{T}+\rightarrow \mathrm{O}_{\mathrm{ISF}}\right)$ and $\mathrm{E}_{\text {seg }}\left(\mathrm{O}(\mathrm{fcc}) \rightarrow \mathrm{T}-{ }_{\mathrm{ISF}}\right)$, contrary to what is reported in [17] using a different $\mathrm{H}$ concentration and relaxation procedure. The same trends in energy are obtained when a separation is applied in the $z$-direction (Figure 4(b) and bottom Table 1). In addition, 4 different meta-stable positions were found for the distorted fcc octahedral site, all with segregation energies in the range 40-70 meV (9-40 meV with ZPE corrections).

\section{Hydrogen diffusion under localised shear}

We now consider hydrogen diffusion, under the effect of $\Delta u_{y}$ and $\Delta u_{z}$. The same geometric distinction is made for diffusion as the one made for segregation: below $\Delta u_{z}=0.5 b$, the structure is considered a sheared fcc structure with diffusion paths connecting distorted $\mathrm{T}+\mathrm{T}-$ and $\mathrm{O}$ sites; beyond $\Delta u_{z}=0.5 \mathrm{~b}$, the structure is considered a distorted hcp structure with paths connecting $\mathrm{T}+$ (hcp), T- (hcp) and $\mathrm{O}$ (hcp). The elementary diffusion jumps are presented on Figure 5(a). The symmetry is broken by the shear in y and jumps along the $y$-direction are distinct from the ones which have a component in $x$. T+ and $\mathrm{T}-$ are equivalent, which leaves four different paths, i.e. six different jumps since $\mathrm{T}+\rightarrow \mathrm{T}-$ jumps are symmetric. From Figure 5(a), they are, for the distorted fcc structure:

- $\mathrm{T}+\leftrightarrow \mathrm{O}(1)$, with an $x$-component

- $\mathrm{T}+\leftrightarrow \mathrm{O}(2)$, without $x$-component

- $\mathrm{T}+\rightarrow \mathrm{T}-(2)$, with an $x$-component

- $\mathrm{T}+\rightarrow \mathrm{T}-(1)$, without $x$-component

and in the distorted hcp structure:

- $\mathrm{O}(\mathrm{h} c \mathrm{p}) \leftrightarrow \mathrm{T}+(\mathrm{hcp})(1)$, with an $x$-component

- $\mathrm{O}(\mathrm{h} c \mathrm{p}) \leftrightarrow \mathrm{T}+(\mathrm{hcp})(2)$, without $x$-component

- $\mathrm{T}+(\mathrm{hcp})(1) \rightarrow \mathrm{T}-(\mathrm{hcp})(3)$, without $x$-component

- $\mathrm{T}+(\mathrm{h} c \mathrm{p})(2) \rightarrow \mathrm{T}-(\mathrm{hcp})(2)$, with an $x$-component

The NEB method is used to calculate the energy barriers relative to the different jumps, at different levels of shear displacement $\Delta u_{y}$. The relaxations are therefore local. We start with the exhaustive results obtained with the EAM interactions. Results obtained with full relaxations and local relaxations, in the undistorted fcc structure and in the ISF structure, are first compared 


$$
\begin{array}{llllll}
\text { Top View } & \begin{array}{lll}
\mathrm{T}+(\mathrm{fcc}) \\
\mathrm{O}(\mathrm{hcp})
\end{array} & \mathrm{T}-(\mathrm{fcc}) & \mathrm{O}(\mathrm{fcc}) \\
& \mathrm{T}+(\mathrm{hcp}) & \mathrm{T}-(\mathrm{hcp})
\end{array}
$$

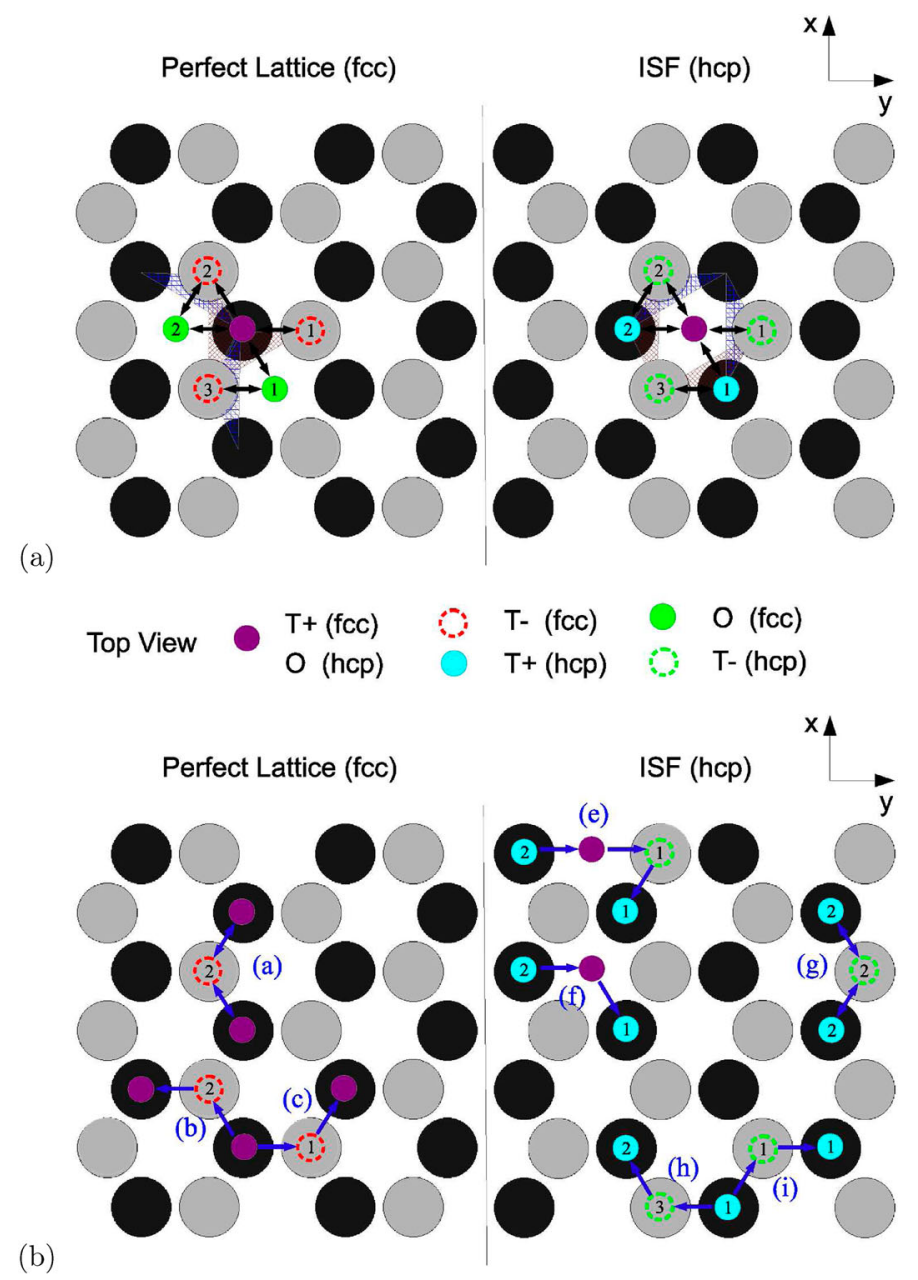

Figure 5. Schematic picture of (a) the elementary jumps and (b) the various diffusion path from a T+ site to another T+ site, in the fcc structure and in the local hcp structure of the ISF, relevant to the EAM potential. The colour code for the different sites is shown on the top of the figure, while the numbers represent different sites of the same nature, in coherence with the enumeration of the diffusion paths in the text.

(Table 2). The differences in energies are less than $0.01 \mathrm{eV}$, which justifies the use of local relaxations for the barriers. An example of energy profiles, obtained by the NEB, for the jumps $\mathrm{T}+\rightarrow \mathrm{T}-(1)$ and $\mathrm{T}+\rightarrow \mathrm{O}(1)$, at two different levels of shear displacement, are given in Figure 6 . The energy barriers for a shear displacement lower than $0.5 b$ are presented in Figure $7(\mathrm{a}, \mathrm{b})$. Note that the deformed $\mathrm{O}$ site is no longer a stable position for $\mathrm{H}$ when the shear is over $0.25 b$. All the barriers decrease with shear. For jumps from the $\mathrm{T}$ site, the easiest jumps are $\mathrm{T}+\leftrightarrow \mathrm{T}-(2)$ and for those involving the $\mathrm{O}$ site they are $\mathrm{O}(2) \rightarrow \mathrm{T}+$. We 
Table 2. Comparison of the barriers computed by full relaxation and local relaxation.

\begin{tabular}{|c|c|c|}
\hline \multirow{2}{*}{ Pathway } & \multicolumn{2}{|c|}{ EAM } \\
\hline & $\mathrm{LR}$ & $\mathrm{FR}$ \\
\hline $\mathrm{T}+\rightarrow 0$ & 0.192 & 0.202 \\
\hline $\mathrm{T}+\rightarrow \mathrm{T}-$ & 0.159 & 0.170 \\
\hline $\mathrm{T}+(\mathrm{hcp}) \rightarrow \mathrm{O}$ (hcp) & 0.156 & 0.159 \\
\hline $\mathrm{T}+(\mathrm{hcp}) \rightarrow \mathrm{T}-(\mathrm{hcp})$ & 0.138 & 0.147 \\
\hline
\end{tabular}

Note: The calculations are performed in the undistorted fcc and hcp (ISF) structures. The unit is eV.

define a hydrogen diffusion step as a sequence of two consecutive jumps which brings $\mathrm{H}$ from a $\mathrm{T}+$ site to another $\mathrm{T}+$ site. The most frequent jumps are those involving jumps from $\mathrm{T}+$ and $\mathrm{T}-$ sites, according to Transition State Theory and since they have the lowest barriers. The easiest jump pathway is therefore pathway (a) through $\mathrm{T}-(2)$ in Figure 5(b). The second easiest are (b) and (c) which involve a $\mathrm{T}+\leftarrow \mathrm{T}-$ jump without a component in $x$ and a second one with a component in $x$. With this potential, whatever the path taken, there is always an acceleration of diffusion due to a fault in the stacking sequence. Furthermore, if one considers an edge Shockley partial, the diffusion velocity will be maximum along the core of the dislocation line, due to path (a). $\mathrm{H}$ on an $\mathrm{O}$ site would jump immediately to neighbouring $\mathrm{T}+/ \mathrm{T}-$ sites due to a low energy barrier connecting these sites.

When the shear is larger than $0.5 b$, the barriers are less affected by the shear, except the $\mathrm{T}+_{\text {ISF }}(1) \rightarrow \mathrm{O}_{\text {ISF }}$ whose barrier increases from 0.02 to $0.16 \mathrm{eV}$ (Figure 7 (c)). There is a cross over with the evolution of the barrier for the $\mathrm{T}+\mathrm{ISF}_{\mathrm{IF}}(2) \leftrightarrow \mathrm{T}$ - ISF (2) jump at about $0.85 b$, a shear upon which this $\mathrm{T} / \mathrm{T}$ jump becomes the preferential diffusion mechanism. Below $0.85 b$, the preferential diffusion path are

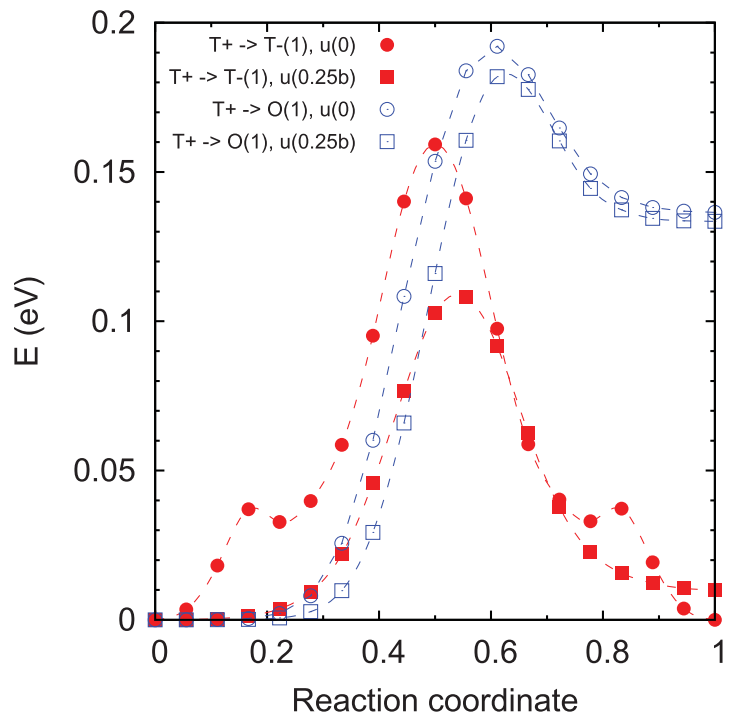

Figure 6. Energy profiles obtained by the NEB for jumps $T+\rightarrow T-(1)$ and $T+\rightarrow O(1)$, at two different levels of shear. The energy barrier is the maximum along this profile. Atomic interactions are given by the EAM potential. 


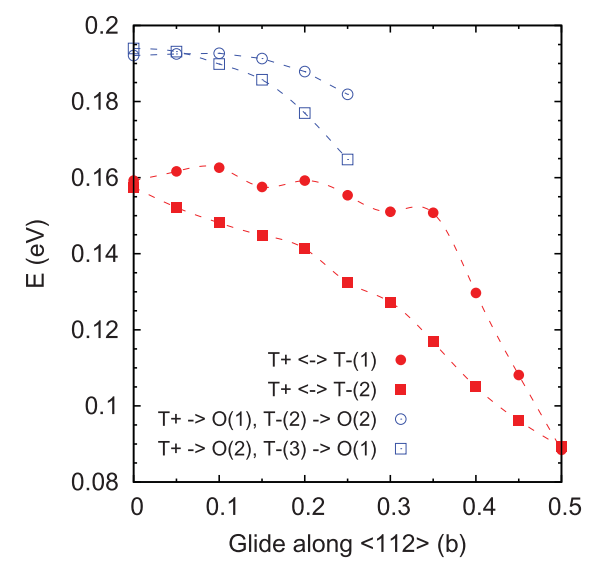

(a)

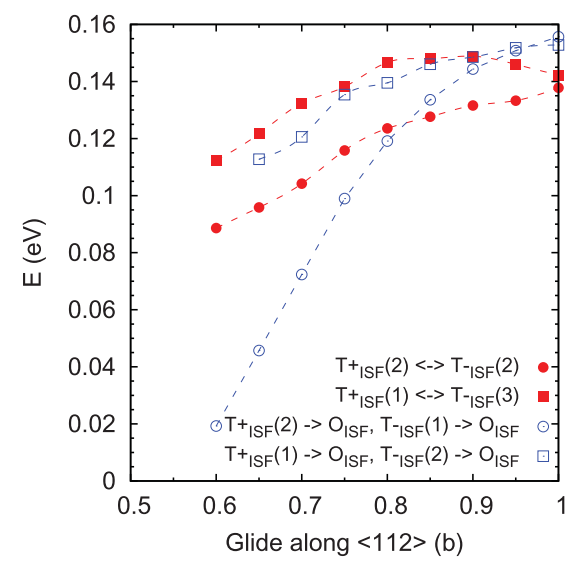

(c)

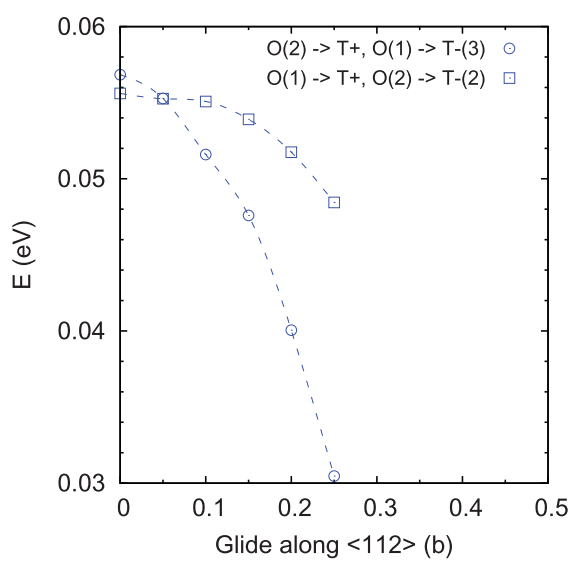

(b)

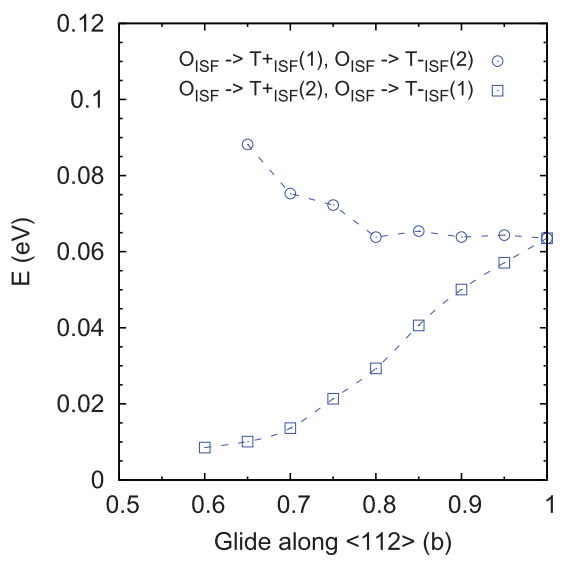

(d)

Figure 7. Evolution of the energy barrier for the various $\mathrm{H}$ jumps as a function of the shear obtained using the EAM potential: (a) for jumps from $T+/ T-$ sites; (b) for jumps from the 0 site; (c) and (d) are similar to (a) and (b) respectively, but with an increase of inter-planar distance $\Delta u_{z}$ on the shear plane.

(e) and (f) (Figure 5(b)). The jumps involve a large displacement component in the shear direction. Beyond $0.85 b$, the diffusion behaviour is similar to the one of the distorted fcc structure. It relies on T/T jumps: path (g) on Figure 5(b), and to a lesser extent on paths (h) and (i). Again, the diffusion would be enhanced along the line direction of an edge Shockley partial.

Some calculations are repeated using DFT. They confirm, essentially, the drop of the activation barrier for diffusion. The results are given in Table 3 . In the undistorted fcc structure, the jump barrier for $\mathrm{T}+\rightarrow \mathrm{O}$ is $0.165 \mathrm{eV}$, which agrees well with other calculations [31,33] and experiments [34]. The segregation calculations reported above indicate that the $T+(\mathrm{fcc})$ site remains the most stable site during the whole shearing process (i.e. it transforms into $\mathrm{O}$ (hcp) which is more stable than $\mathrm{T}$ (hcp)). The shear induced reduction in the 
Table 3. Hydrogen jump barriers, computed by DFT, with local relaxations (eV).

\begin{tabular}{|c|c|c|c|}
\hline$\left(\Delta u_{y}, \Delta u_{z}\right)$ & $(0,0)$ & $(0.45 b, 0)$ & $(1 b, 0)$ \\
\hline $\mathrm{T}+\rightarrow 0$ & $\begin{array}{l}0.165^{\mathrm{a}} \\
0.17[33] \\
0.15^{\mathrm{b}}, 0.18[31] \\
0.16_{\exp }[34]\end{array}$ & & \\
\hline $\mathrm{T}+\rightarrow \mathrm{O}_{2}$ & & $\begin{array}{l}0.143 \\
0.142^{b}\end{array}$ & \\
\hline $\mathrm{T}+\rightarrow \mathrm{O}_{1}$ & & $\begin{array}{l}0.093 \\
0.049^{b}\end{array}$ & \\
\hline $\mathrm{O}_{I S F} \rightarrow \mathrm{T}_{\text {ISF }}(2)$ & & & $\begin{array}{l}0.118 \\
0.164^{b}\end{array}$ \\
\hline $\mathrm{O}_{I S F} \rightarrow \mathrm{T}+I S F(1)$ & & & $\begin{array}{l}0.123 \\
0.169^{b}\end{array}$ \\
\hline$\left(\Delta u_{y}, \Delta u_{z}\right)$ & $\left(0,0.05 a_{0}\right)$ & $\left(0.45 b, 0.05 a_{0}\right)$ & $\left(1 b, 0.05 a_{0}\right)$ \\
\hline $\mathrm{T}+\rightarrow \mathrm{O}_{1}$ & 0.102 & $\begin{array}{l}0.088 \\
0.047^{b}\end{array}$ & \\
\hline $\mathrm{O}_{I S F} \rightarrow \mathrm{T}+$ ISF $(1)$ & & & $\begin{array}{l}0.059 \\
0.042^{b}\end{array}$ \\
\hline
\end{tabular}

Note: The decrease of the activation energy found with the EAM when the interstitial sites are sheared is confirmed. The dilatation perpendicular to the glide plane $\Delta u_{z}$ also decreases the jump barriers.

${ }^{a}$ Full relaxation.

${ }^{b}$ ZPE correction included.

jump barrier is confirmed: the barrier for pathway $\mathrm{T}+\leftrightarrow \mathrm{O}(1)$ decreases by about $66 \%$ at a shear level of $0.45 b$ (Table 1). Half of this drop comes from the ZPE. The $\mathrm{T}+\leftrightarrow \mathrm{T}-$ jumps are associated with two imaginary frequencies and are not considered further. On the contrary, it is found that in the ISF structure the migration barriers are very similar to the bulk ones. Therefore, there is only an acceleration of diffusion in the core of the dislocations. In particular, since the jump $\mathrm{T}+\leftrightarrow \mathrm{O}(1)$ has a component in $\mathrm{x}$, the DFT calculations indicate the possibility of pipe diffusion at edge dislocation cores. The order of magnitude of the barrier decrease is of $0.1 \mathrm{eV}$. Transition State Theory gives a boost in the jump rate, at room temperature, of $\exp 0.1 / k T \sim 50$. Modelling the impact on diffusion requires taking also into account the segregation in the stacking fault [35]. This behaviour is very similar to what was found in another fcc metal, Pd [10] where the core sites also offer a fast diffusion track (the jump barrier is also decreased by about $50 \%$ ).

In addition, the effect of a displacement perpendicular to the shearing direction $\left(\Delta u_{z}\right)$ has been quantified. It represents the effect of a mode I loading on the sub-surface interstitial sites [20]. The site preference is not modified (Table 1). The traction involves a moderate segregation towards the shear plane $\left(E_{\text {seg }} \sim-50 \mathrm{meV}\right)$. Similar to the case without traction, the diffusion barriers are largely decreased (Table 3).

\section{Discussion}

Two results can be specifically discussed with respect to the literature concerning $\mathrm{H}$ embrittlement: the impact of $\mathrm{H}$ segregation on the intrinsic staking fault (ISF) 
energy and the role played by dislocations in $\mathrm{H}$ trapping. First, there has been a debate $[1,36]$ about the influence of $\mathrm{H}$ on the 'mobility' of dislocations. It was proposed, based on in situ transmission microscopy, that $\mathrm{H}$ reduces the elastic interaction between a dislocation and the obstacles in the glide plane resulting in an enhanced depinning [1]. At the same time, dislocations have to drag the Cotrell atmosphere composed of segregated $\mathrm{H}$ atoms which tend to pin them. The same elastic shielding, but of the stress field created by the dislocation itself, can reduce the line tension [4] with consequences on dislocation multiplication. Another aspect of the 'Hydrogen Enhanced Localized Plasticity' model is slip planarity. It is classically related to the intrinsic stacking fault energy [4] with constriction mechanisms of the core necessary for dislocations to change the glide plane. Then materials with a high (resp. low) ISF energy have high probabilities for cross slip events (resp. low). One possible mechanism for an $\mathrm{H}$ enhanced slip planarity is a decrease of the cross slip probability due to a decrease in the ISF energy. From the segregation energy $\Delta E_{s e g}=-0.066 \mathrm{eV}$ in Table 1 and the ISF energy $\gamma_{I S F}=120 \mathrm{~mJ} / \mathrm{m}^{2}$ [20] (the LDA approximation gives the value of $165 \mathrm{~mJ} / \mathrm{m}^{2}$ [37]) the variation of the ISF energy with bulk hydrogen concentration $c_{b u l k}$ is given by

$$
\begin{gathered}
\gamma_{I S F}\left(c_{b u l k}\right)=\gamma_{I S F}+c \times \frac{\Delta E_{s e g}}{S_{a t}} \\
\frac{c}{1-c}=\frac{c_{b u l k}}{1-c_{b u l k}} \exp \frac{-\Delta E_{s e g}}{k T}
\end{gathered}
$$

where $S_{a t}=\sqrt{3} / 4 a_{0}^{2}$ is the surface per octahedral site, in the $\{111\}$ orientation and $c$ is the average occupancy of an octahedral site in the ISF in equilibrium with a bulk concentration $c_{\text {bulk }}$ on tetrahedral sites at temperature $T . k$ is the Boltzmann constant. The reduction of $\gamma_{I S F}$ is very significant but only at very high concentrations, beyond $1 \%$ atomic (Figure 8 ). Two curves are added for comparison: Al with $\Delta E_{\text {seg }}$ decreased down to $-0.1 \mathrm{eV}$ (to test the robustness of the message with respect to an effect of a superimposed hydrostatic stress from a partial dislocation line) and Pd with $\Delta E_{\text {seg }}=-0.044 \mathrm{eV}$ [38].

Second, it has been measured that heavy plastic deformation decreases the effective diffusion coefficient (see Figure 7 in [39] and also [40]). At the microscopic level, possible reasons for this decrease are: hydrogen trapping in the dislocation elastic stress field (especially the hydrostatic tension in the vicinity of the line of the edge dislocation [41]) and trapping at the dislocation core. The trapping could also come from vacancies or internal stresses produced by the heavy plastic deformation [40]. In this section, the influence of the trapping at the stacking fault, or in the elastic field, on the effective diffusion coefficient and the possibility to have 'pipe diffusion' along the dislocation line is quantified. Following [39,42], the effective diffusion coefficient $D_{\text {eff }}$, considering a hydrogen atom performing a long, 


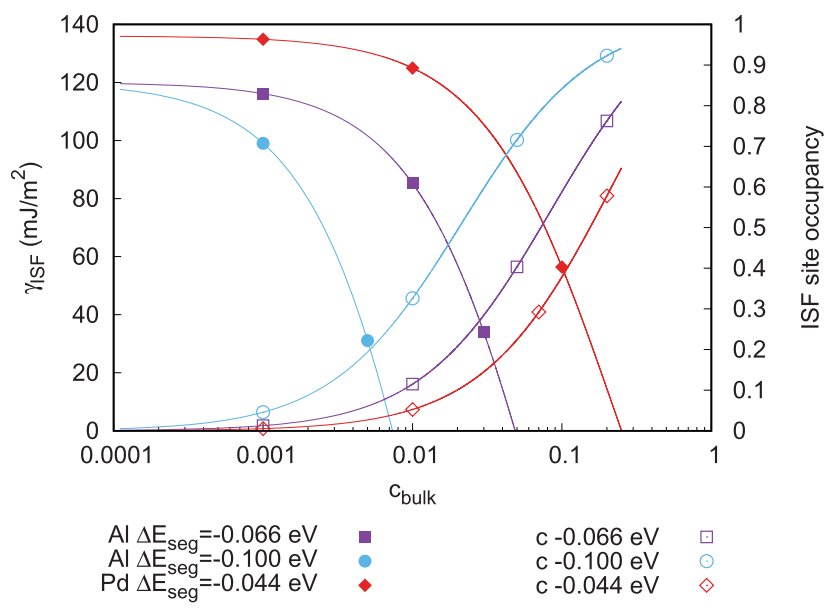

Figure 8. Evolution of the stacking fault energy $\gamma_{I S F}$ with the bulk hydrogen concentration at $T=$ $300 \mathrm{~K}$ according to Equation 2. Additional curves represent the case of $\mathrm{Pd}$ and $\mathrm{Al}$ with a decreased segregation energy to test the robustness of the trend. Occupancies $c$ of the octahedral site in the ISF is also given (Equation 3).

uncorrelated, random walk among regular lattice sites and trap sites, at equilibrium, is given by the Einstein formula:

$$
D_{\text {eff }}=\frac{1}{2 d} \lim _{N \rightarrow \infty} \sum_{n=0}^{N} \frac{\Delta \vec{r}_{n}^{2}}{t}
$$

where $N$ is the number of jumps, $t$ is the corresponding time and $\Delta \vec{r}_{n}$ is the displacement vector of the $n$th jump. The sum can be rearranged by grouping the terms that correspond to each specific type of jumps:

$$
D_{e f f}=\frac{1}{2 d} \lim _{N \rightarrow \infty} \sum_{i} \sum_{j} \frac{N_{i j}}{t_{i}} \frac{t_{i}}{t} \Delta \vec{r}_{i j}^{2}
$$

where $i$ is the index of the tetrahedral site, $j$ is the index of the transition, starting at site $i, N_{i j}$ is the number of transitions $i j$ occurring during time $t_{i}$ and $t_{i}$ is the amount of time the $\mathrm{H}$ atom spends on site $i$ during time $t$. $\Delta \vec{r}_{i j}^{2}$ is the (squared) amplitude of the jump $i j$. In the limit $N \rightarrow \infty, N_{i j} / t_{i}$ is the rate of the jump $i j \Gamma_{i j}$ and $t_{i} / t$ is the mean occupancy of site $i \overline{p_{i}}$. Then the effective diffusion coefficient is

$$
D_{e f f}=\frac{1}{2 d} \sum_{i} \overline{p_{i}} \sum_{j} \Gamma_{i j} \Delta \vec{r}_{i j}^{2}
$$

$\mathrm{H}$ diffusion jumps are considered as Poisson processes with rates $\Gamma_{i j}$ given by

$$
\Gamma_{i j}=v_{0} \exp -E_{a}^{i j} / k T
$$

with the activation barrier $E_{a}^{i j}$ being the energy difference between the saddle 
configuration and the minimum energy configuration

$E_{a}^{i j}=E_{\text {saddle }}^{i j}-E_{\text {minimum }}^{i}$. For an edge dislocation, the sum in Equation (6) can be split into three parts. The first one contains the contribution of the sites only affected by the elastic deformation. In the second one, the sites belong to the stacking fault. There, trapping occurs with $\Delta E_{\text {seg }}^{i}=-0.066$ $\mathrm{eV}$ but the diffusion barriers are similar to the one in the bulk (no pipe diffusion). The third part contains the contribution of the sites of the dislocations which border the stacking fault. At this location, there is no trapping $\left(\Delta E_{\text {seg }}^{i} \approx 0\right)$ but pipe diffusion occurs (the barriers are lowered in the direction of the line). Let us evaluate the elastic effect first. Hydrogen is sensitive to hydrostatic stress fields, to which the segregation energy is simply proportional. Following [41], the segregation energy due to the elastic field is written as

$$
\Delta E_{\text {seg }}^{i}=\beta \frac{\theta_{i}}{r_{i}}
$$

where $\theta_{i}$ and $r_{i}$ are the polar coordinates defined in [41] with the extra plane of the edge dislocation above the glide plane and $\beta=\left(\mu b_{e} / 3 \pi\right)((1+v) /(1-v) \Delta v)$ where $\mu$ and $v$ are the elastic constants, $b_{e}$ is the norm of the Burgers vector and $\Delta v$ represents a local volume change related to the insertion of the $\mathrm{H}$ atom at position $\left(\theta_{i}, r_{i}\right)$. With $\Delta v$ equal to $20 \%$ of the atomic volume, the segregation energy at $\left(\theta_{i}=-\pi / 2, r_{i}=b_{e}\right)$ is $-0.13 \mathrm{eV}$, a reasonable value for searching an upper bound of the elastic trapping effect (for DFT calculations on $\mathrm{H}$ at a screw dislocation in Fe, see [44]). Furthermore, stress also affects the energy in the saddle configuration, also in a linear way [43]. The same form as Equation (8) is chosen for the variation the saddle energy $\Delta E_{\text {saddle }}^{i j}=\alpha \beta \theta_{i} / r_{i}$ with a proportionality factor $\alpha$. The $a b$ initio calculations in [17] give a drop of the activation barrier of $-0.0056 \mathrm{eV}$ every \% of hydrostatic strain. It corresponds to a factor $\alpha=0.11507$. To give an order of magnitude, the strain in $\left(\theta_{i}=-\pi / 2, r_{i}=b_{e}\right)$ is of the order of $2.6 \%$ and the corresponding drop of the barrier energy is $-0.015 \mathrm{eV}$ (the barrier without stress is $0.15 \mathrm{eV}$ ). Now the effective diffusion coefficient related to the elastic stress effect alone can be calculated from Equation (6). The probability, at equilibrium, that a hydrogen atom occupies site $i$ is:

$$
\overline{p_{i}}=\frac{\exp \left(-\Delta E_{\text {seg }}^{i} / k T\right)}{\sum_{i} \exp \left(-\Delta E_{\text {seg }}^{i} / k T\right)}
$$

where the extent of the sum depends on the dislocation density. For this, a thin layer of width one period along the dislocation line and a square surface centred on it is considered such that the number of sites $M$ over which them 
sum in Equation (6) runs is related to the dislocation density by $1 / \rho=M a_{0}^{2} / 4$ for the fcc crystal oriented with respect to an edge dislocation (the periodic cell has dimensions $\sqrt{2} / 2 a_{0} \times \sqrt{3 / 2} a_{0} \times \sqrt{3} a_{0}$, contains 6 atoms and the dislocation lies along $y$ ). The activation barrier is modified by the stress such that $E_{a}^{i j}=E_{\text {saddle }}^{0}+\Delta E_{a}^{i j}-E_{\text {minimum }}^{0}-\Delta E_{\text {seg }}^{i}$, and $E_{a}=E_{\text {saddle }}^{0}-E_{\text {minimum }}^{0}$ is the activation barrier without stress. The effective diffusion coefficient is

$$
\begin{aligned}
D_{\text {eff }}^{\text {elastic }}= & \frac{1}{2 d} \sum_{i}\left\{\frac{\exp \left(-\Delta E_{\text {seg }}^{i} / k T\right)}{\sum_{i} \exp \left(-\Delta E_{\text {seg }}^{i} / k T\right)}\right. \\
& \left.\sum_{j} \nu_{0} \exp -\left(E_{a}+\Delta E_{a}^{i j}-\Delta E_{\text {seg }}^{i}\right) / k T \Delta \vec{r}_{i j}^{2}\right\} \\
= & D_{0} \frac{\sum_{i} 1 / 4 \sum_{j} \exp \left(-\alpha \beta \theta_{i j} / r_{i j} k T\right)}{\sum_{i} \exp \left(-\beta \theta_{i} / r_{i} k T\right)}
\end{aligned}
$$

where $\left(\theta_{i j}, r_{i j}\right)$ is the coordinate at the half jump position. The ratio $D_{\text {eff }}^{\text {elastic }} / D_{0}$ is presented on Figure 9 , as a function of the dislocation density. The elastic trapping is negligible at common dislocation densities (below $10^{15} \mathrm{~m}^{-2}$ ) and is therefore ignored in the next calculations.

The system is now considered to be composed of $N_{b u l k}$ bulk sites, $N_{S F}$ sites of the stacking fault (core sites) and $N_{\text {line }}$ sites of the dislocation lines. Equation (6)

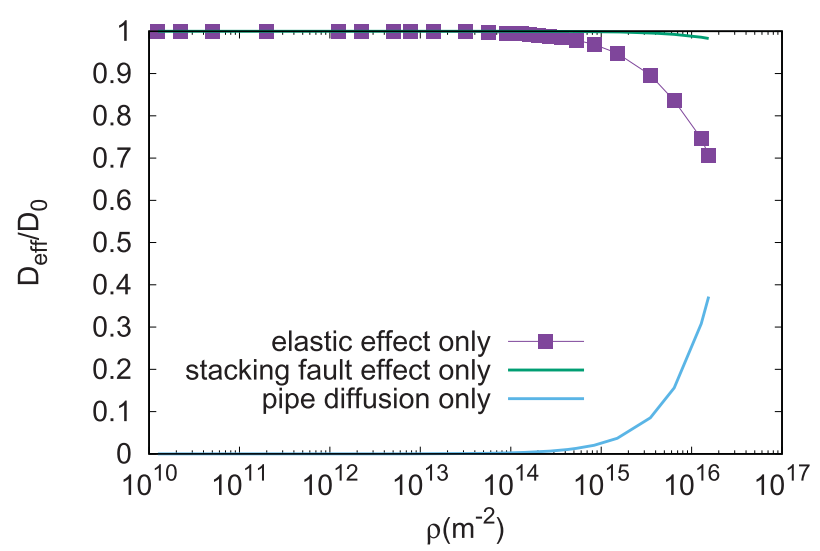

Figure 9. Evolution of the ratio between the effective diffusion coefficient and the bulk diffusion coefficient as a function of the density of dislocations $\rho$. Temperature is $300 \mathrm{~K}$. The segregation to the stacking fault is $-0.066 \mathrm{eV}$. The effect of pipe diffusion corresponds to a boost of 300 (see the text). 
gives, ignoring the elastic effects:

$$
\begin{aligned}
& D_{\text {eff }}=\frac{1}{2 d}\left(\frac{1}{1+\frac{N_{S F}}{N_{\text {bulk }}} \exp -\left(\Delta E_{\text {seg }} / k T\right)+\frac{N_{\text {line }}}{N_{\text {bulk }}}} \sum_{j} \Gamma_{0} \Delta r_{0}^{2}\right. \\
& +\frac{\frac{N_{S F}}{N_{\text {bulk }}} \exp -\left(\Delta E_{\text {seg }} / k T\right)}{1+\frac{N_{S F}}{N_{\text {bulk }}} \exp -\left(\Delta E_{\text {seg }} / k T\right)+\frac{N_{\text {line }}}{N_{\text {bulk }}}} \sum_{j} \Gamma_{0} \exp \left(\Delta E_{\text {seg }} / k T\right) \Delta r_{0}^{2} \\
& \left.+\frac{\frac{N_{\text {line }}}{N_{\text {bulk }}}}{1+\frac{N_{S F}}{N_{\text {bulk }}} \exp -\left(\Delta E_{\text {seg }} / k T\right)+\frac{N_{\text {line }}}{N_{\text {bulk }}}} \sum_{j} \Gamma_{i j} \Delta r_{i j}^{2}\right)
\end{aligned}
$$

It is assumed that the barrier to exit from the stacking fault is $E_{a}-\Delta E_{\text {seg }}$ which leads to a compensation and finally

$$
\begin{aligned}
D_{\text {eff }} / D_{0}= & \frac{1}{1+\frac{N_{S F}}{N_{\text {bulk }}} \exp -\left(\Delta E_{\text {seg }} / k T\right)+\frac{N_{\text {line }}}{N_{\text {bulk }}}} \\
& +\frac{N_{\text {bulk }}}{1+\frac{N_{S F}}{N_{\text {bulk }}} \exp -\left(\Delta E_{\text {seg }} / k T\right)+\frac{N_{\text {line }}}{N_{\text {bulk }}}} \\
& +\frac{1}{2 d D_{0}} \frac{N_{\text {line }}}{1+\frac{N_{S F}}{N_{\text {bulk }}} \exp -\left(\Delta E_{\text {seg }} / k T\right)+\frac{N_{\text {line }}}{N_{\text {bulk }}}} \sum_{j} \Gamma_{i j} \Delta r_{i j}^{2}
\end{aligned}
$$

The first term in Equation (12) is the Oriani model [42,45], the second term is the negligible contribution from the sites of the stacking fault themselves $\left(N_{S F}<<N_{b u l k}\right)$ and the third term is the contribution from the pipe diffusion along the line. The trapping from the stacking fault has a very small effect (Figure 9). The same methodology [46] as the one used for modelling vacancy effects [42] on hydrogen diffusion is now used to evaluate the contribution from pipe diffusion. The model considers the diffusion along the dislocation line as a one dimensional random walk with a 'leak', i.e. after entering the dislocation line, a hydrogen atom performs a sequence of fast $\mathrm{T} \rightarrow \mathrm{T}$ jumps along the line, with the possibility of rare 'escape from the line' jumps. In order to simplify the calculation, the intermediate octahedral sites are not considered. The goal is to evaluate $\sum_{j} \Gamma_{i j} \Delta r_{i j}^{2}$, where $i$ is the entrance site in the dislocation line and $j$ is the exit site, at a (squared) distance $\Delta r_{i j}^{2}=3 / 8 \Delta r_{0}^{2} *(i-j)^{2}$ if $(i-j)$ is the number of $\mathrm{T}$ sites inbetween the entrance and exit sites and $\Delta r_{0}$ 
is the amplitude of a $\mathrm{T} \rightarrow \mathrm{O}$ jump. The calculation requires the evaluation of the time evolution of the occupancy $o_{i}$ of the various sites. This is obtained by solving the master equations $[42,46]$ :

$$
\begin{gathered}
\frac{\mathrm{d} o_{i}}{\mathrm{~d} t}=o_{i-1}(t) \Gamma_{i-1 i}+o_{i+1}(t) \Gamma_{i+1}-o_{i}(t)\left(\Gamma_{i-1}+\Gamma_{i+1}\right)-o_{i}(t) \sum_{k} \Gamma_{i k}^{e x i t} \\
\frac{\mathrm{d} o_{\text {out }}}{\mathrm{d} t}=\sum_{i} \sum_{k} o_{i}(t) \Gamma_{i k}^{\text {exit }}
\end{gathered}
$$

with $\Gamma_{i+1}=v_{0} \exp \left(-E_{\text {line }} / k T\right)\left(E_{\text {line }}=0.05 \mathrm{eV}\right.$ from Table 3 for $\Delta u_{y}=0.45$ b) and $\Gamma_{i k}^{\text {exit }}=v_{0} \exp \left(-E_{a} / k T\right)=\Gamma_{0}\left(E_{a}=0.15 \mathrm{eV}\right.$ from Table $\left.3 \Delta u_{y}=0\right)$. The number of exit paths per site of the line is 8 taking into account the two $\mathrm{T} \rightarrow \mathrm{O}$ pathes and the $6 \mathrm{O} \rightarrow \mathrm{T}$ pathes which brings the $\mathrm{H}$ outside the dislocation line. $o_{\text {out }}$ measures the probability that $\mathrm{H}$ has exited the line, whatever the exit path it took. The numerical resolution (Figure 10) requires that a finite number of sites along the line is considered and therefore periodic boundary conditions are used. At time 0 , a hydrogen atom enters the line at site $i$, i.e. the initial conditions for the master equation are $o_{i}(0)=1$ and $o_{j}(0)=0$. The probability that the hydrogen atom exits from site $j$, after entering at site $i$, in between times $\mathrm{t}$ and $\mathrm{t}+\mathrm{dt}$ is $o_{j}(t) \sum_{k} \Gamma_{j k}^{e x i t} d t=o_{j}(t) \times 8 \Gamma_{0} d t$ and the probability that the $\mathrm{H}$ exits by site $\mathrm{j}$ is $\int_{0}^{\infty} o_{j}(t) \times 8 \Gamma_{0} d t$. This probability is easy to calculate from the eigenvalues and eigenvectors of the transition rate matrix [46] corresponding to Equations (13) and (14). The acceleration of diffusion due to pipe

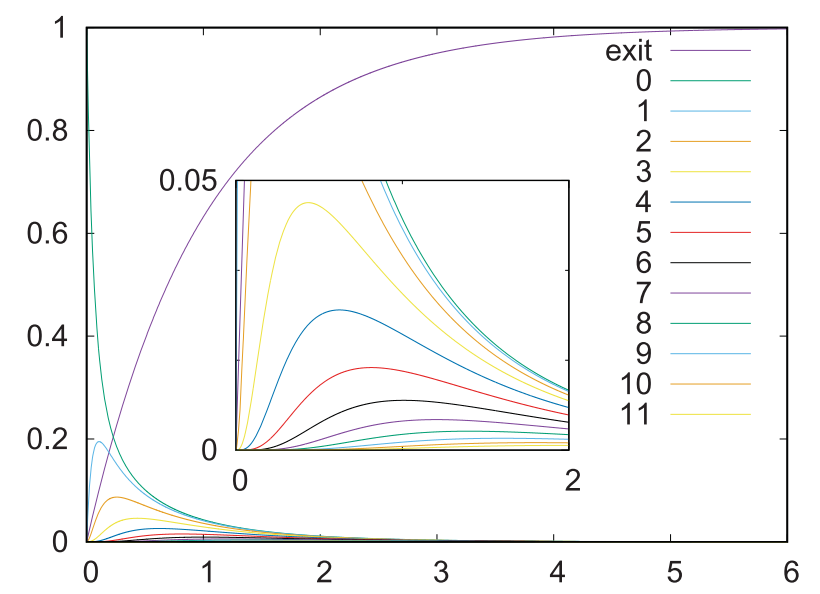

Figure 10. Time evolution of the $o_{i}$ given by Equations (13) and (14) $\left(T=300 \mathrm{~K}\right.$, time in $\left.1 /\left(8 \Gamma_{0}\right)\right)$. $\mathrm{H}$ enters the dislocation line by site 0 . The numbers are the index of the sites, numbered with respect to the entrance site 0 . 
diffusion is

$$
\begin{aligned}
\frac{1}{2 \mathrm{~d} D_{0}} \sum_{j} \Gamma_{i j} \Delta r_{i j}^{2} & =\frac{1}{2 \mathrm{~d} D_{0}} \sum_{j} j^{2} \int_{0}^{\infty} o_{j}(t) \times 8 \Gamma_{0} d t \times 3 / 8 \Delta r_{0}^{2} 8 \Gamma_{0} \\
& =3 / 4 \sum_{j} j^{2} \int_{0}^{\infty} o_{j}(t) \times 8 \Gamma_{0} \mathrm{~d} t
\end{aligned}
$$

At $T=300 \mathrm{~K}$, this boost factor is 8.9 , while at $T=150 \mathrm{~K}$, it is 300 (or, equivalently, if a system where the barriers were doubled i.e. 0.3 and $0.1 \mathrm{eV}$ is considered). Inserted in Equation 12, this result has no effect on macroscopic diffusion, because the probability that the $\mathrm{H}$ atom enters the dislocation line is too small at the dislocations densities considered. Only the larger boost of 300 could have an effect, but even in this case it would just counter balance the elastic trapping effect, at high dislocation density (Figure 9). The conclusion of the quantification of the trapping/accelerating effects of dislocations is that the impact on diffusion, at the macroscopic scale, is weak. The hypothesis of a massive trapping due to the debris produced by straining [47] remains. Indeed, the hydrogen binding to vacancies is strong enough in $\mathrm{Al}$ that $1 \mathrm{ppm}$ vacancies decreases the diffusion coefficient by a factor of 10 [48]. Note that, at the nanoscale, both the trapping and pipe diffusion phenomena were observed by tritium autoradiography in TEM, in Al [49]. This is coherent with the calculations presented here because it is only the low density of defects which hides the effects at the macroscale.

\section{Conclusion}

As a summary, this paper studies the effect of a localised shear in a glide plane, typically in the core of an edge dislocation, on hydrogen stability and migration. DFT calculations show that the tetrahedral site of the face centred cubic structure of $\mathrm{Al}$ remains the preferential hosting site for $\mathrm{H}$. It continuously transforms into the octahedral site of the local hexagonal compact structure of the intrinsic stacking fault. No stability crossover with the octahedral site was found. The energy difference between the two increases significantly in the ISF. Furthermore, diffusion barrier calculations show that the imperfect stacking significantly enhances diffusion, but not in the ISF where it is similar to the one in the bulk. Diffusion becomes an-isotropic, with a marked reduction of barriers perpendicular to the Burgers vector, i.e. a tendency to pipe diffusion along edge Shockley dislocation cores. The order of magnitude of the boost on the jump rate is a factor 50 , at room temperature. The effects of elastic and core segregation on the macroscopic effective diffusion coefficient show that the effect is too weak, at the usual dislocation densities, to be detected. Pipe diffusion is also 
quantified by First Passage Time Analysis [46] and, for the same reason, has a very weak effect at the macroscopic scale, for Al, although coherent with nanoscale experiments [49]. In addition, the $\mathrm{Al}-\mathrm{H}$ inter-atomic potential described in [24] has been extensively tested, which will be useful for further studies of subsurface $\mathrm{H}$ effects on dislocation emission at crack tips [20].

\section{Disclosure statement}

No potential conflict of interest was reported by the authors.

\section{Funding}

This work was performed using HPC resources from CALMIP (Grant 2018-p0749) and Agence Nationale de la Recherche (Blanc 10- 19424).

\section{References}

[1] I.M. Robertson, P. Sofronis, A. Nagao, M.L. Martin, S. Wang, D.W. Gross, and K.E. Nygren, Hydrogen embrittlement understood, Metall. Mater. Trans. A 46A (2015), pp. 2323-2341.

[2] M.L. Martin, B.P. Somerday, R.O. Ritchie, P. Sofronis, and I.M. Robertson, Hydrogeninduced intergranular failure in Nickel revisited, Acta. Mater. 60 (2012), pp. 2739-2745.

[3] I.M. Robertson, The effect of hydrogen on dislocation dynamics, Eng. Fract. Mech. 68 (2001), pp. 671-692.

[4] G. Girardin, C. Huvier, D. Delafosse, and X. Feaugas, Correlation between dislocation organization and slip bands: TEM and AFM investigations in hydrogen-containing nickel and nickel-chromium, Acta Mater. 91 (2015), pp. 141-151.

[5] I. Aubert, N. Saintier, J.M. Olive, and F. Plessier, A methodology to obtain data at the slip-band scale from atomic force microscopy observations and crystal plasticity simulations, Acta Mater. 104 (2016), pp. 9-17.

[6] R.P. Gangloff, H-enhanced deformation and fracture in the crack tip process zone, in Materials Performance in Hydrogen Environments: Proceedings of the 2016 International Hydrogen Conference, 2017, pp. 1-35.

[7] D.N. Ilin, N. Saintier, J.M. Olive, R. Abgrall, and I. Aubert, Simulation of hydrogen diffusion affected by stress-strain heterogeneity in polycrystalline stainless steel, Int. J. Hydrog. Energy 39 (2014), pp. 2418-2422.

[8] A.H.M. Krom, R.W.J. Koers, and A. Bakker, Hydrogen transport near a blunting crack tip, J. Mech. Phys. Solids 47 (1999), pp. 971-992.

[9] P. Sofronis and R. McMeeking, Numerical analysis of hydrogen transport near a blunting crack tip, J. Mech. Phys. Solids 37 (1989), pp. 317-350.

[10] B.J. Heuser, D.R. Trinkle, N. Jalarvo, J. Serio, E.J. Schiavone, E. Mamontov, and M. Tyagi, Direct measurement of hydrogen dislocation pipe diffusion in deformed polycrystalline Pd using quasielastic neutron scattering, Phys. Rev. Lett. 113 (2014), p. 025504.

[11] D. Di Stefano, M. Mrovec, and C. Elsässer, First-principles investigation of hydrogen trapping and diffusion at grain boundaries in nickel, Acta Mater. 98 (2015), pp. 306-312.

[12] H. Vehoff and W. Rothe, Gaseous hydrogen embrittlement in FeSi and Ni single crystals, Acta Metall. 31 (1983), pp. 1781-1793. 
[13] S.P. Lynch, Mechanisms and kinetics of environmentally assisted cracking: Current status, issues, and suggestions for further work, Metall. Mater. Trans. A 44 (2013), pp. 1209-1229.

[14] Y. Sun, Q. Peng, and G. Lu, Quantum mechanical modeling of hydrogen assisted cracking in aluminum, Phys. Rev. B 88 (2013), p. 104109.

[15] R.J. Zamora, A.K. Nair, R.G. Hennig, and D.H. Warner, Ab initio prediction of environmental embrittlement at a crack tip in aluminum, Phys. Rev. B 86 (2012), p. 060101.

[16] V.V. Bulatov and E. Kaxiras, Semidiscrete variational Peierls framework for dislocation core properties, Phys. Rev. Lett. 78 (1997), p. 4221.

[17] G. Lu, D. Orlikowski, I. Park, O. Politano, and E. Kaxiras, Energetics of hydrogen impurities in aluminum and their effect on mechanical properties, Phys. Rev. B 65 (2002), p. 064102.

[18] J.R. Rice, Dislocation nucleation from a crack tip: an analysis based on the Peierls concept, J. Mech. Phys. Solids 40 (1992), pp. 239-271.

[19] F. Apostol and Y. Mishin, Hydrogen effect on shearing and cleavage of Al: A first-principles study, Phys. Rev. B 84 (2011), p. 104103.

[20] Y. Wang, D. Connétable, and D. Tanguy, Sub-surface hydrogen effect on crack tip plasticity in aluminum, to be published (2018).

[21] K. Gouriet and D. Tanguy, Dislocation emission from a crack under mixed mode loading studied by molecular statics, Philos. Mag. 92 (2012), pp. 1663-1679.

[22] V. Vítek, Intrinsic stacking faults in body-centred cubic crystals, Philos. Mag. 18 (1968), pp. 773-786.

[23] S.J. Zhou, A.E. Carlsson, and R. Thomson, Dislocation nucleation and crack stability: Lattice green's-function treatment of cracks in a model hexagonal lattice, Phys. Rev. B 47 (1993), pp. 7710-7719.

[24] X.J. Shen, D. Tanguy, and D. Connétable, Atomistic modelling of hydrogen segregation to the $\sigma 9\{221\}[110]$ symmetric tilt grain boundary in Al, Philos. Mag. 94 (2014), pp. $2247-2261$.

[25] G. Kresse and J. Hafner, Ab initio molecular dynamics for liquid metals, Phys. Rev. B 47 (1993), p. 558.

[26] G. Kresse and J. Hafner, Ab initio molecular-dynamics simulation of the liquid-metalamorphous-semiconductor transition in germanium, Phys. Rev. B 49 (1994), p. 14251.

[27] G. Kresse and J. Furthmüller, Efficient iterative schemes for ab initio total-energy calculations using a plane-wave basis set, Phys. Rev. B 54 (1996), p. 11169.

[28] J.P. Perdew, K. Burke, and M. Ernzerhof, Generalized gradient approximation made simple, Phys. Rev. Lett. 77 (1996), pp. 3865-3868.

[29] G. Kresse and D. Joubert, From ultrasoft pseudopotentials to the projector augmentedwave method, Phys. Rev. B 59 (1999), p. 1758.

[30] H. Monkhorst and J. Pack, Special points for Brillouin-zone integrations, Phys. Rev. B 13 (1976), p. 5188.

[31] C. Wolverton, V. Ozolins, and M. Asta, Hydrogen in aluminum: First-principles calculations of structure and thermodynamics, Phys. Rev. B 69 (2004), p. 144109.

[32] H. Jónsson, G. Mills, and K. Jacobsen, Classical and Quantum Dynamics in Condensed Phase Simulations, World Scientific, Singapore, 1998.

[33] L. Ismer, M.S. Park, A. Janotti, and C.V. de Walle, Interactions between hydrogen impurities and vacancies in $\mathrm{Mg}$ and Al: A comparative analysis based on DFT, Phys. Rev. B 80 (2009), p. 184110.

[34] G.A. Young Jr and J.R. Scully, The diffusion and trapping of hydrogen in high purity aluminum, Acta Mater. 46 (1998), pp. 6337-6349. 
[35] D.N. Ilin, A.A. Kutsenko, D. Tanguy, and J.M. Olive, Effect of grain boundary trapping kinetics on diffusion in polycrystalline materials: Hydrogen transport in Ni, Model. Simul. Mater. Sci. Eng. 24 (2016), p. 035008.

[36] J. Song and W. Curtin, Mechanisms of hydrogen-enhanced localized plasticity: An atomistic study using Fe as a model system, Acta Mater. 68 (2014), pp. 61-69.

[37] Y. Sun and E. Kaxiras, Slip energy barriers in aluminium and implications for ductilebrittle behaviour, Phil. Mag. A 75 (1997), pp. 1117-1127.

[38] B. Amin-Ahmadi, D. Connétable, M. Fivel, D. Tanguy, R. Delmelle, S. Turner, L. Malet, S. Godet, T. Pardoen, J. Proost, and D. Schryvers, Dislocation/hydrogen interaction mechanisms in hydrided nanocrystalline palladium films, Acta Mater. 111 (2016), pp. 253-261.

[39] R. Kirchheim, Solid solutions of hydrogen in complex materials, Solid State Phys. 59 (2004), pp. 203-305.

[40] E.V. den Eeckhout, A. Laureys, Y.V. Ingelgem, and K. Verbeken, Hydrogen permeation through deformed and heat-treated ARMCO pure iron, Mat. Sci. Tech. 33 (2017), pp. $1515-1523$.

[41] J.P. Hirth and J. Lothe, Theory of Dislocations, Krieger Publishing Company, 1992.

[42] Y. Wang, D. Connétable, and D. Tanguy, Influence of trap connectivity on $H$ diffusion: Vacancy trapping, Acta Mater. 103 (2016), pp. 334-340.

[43] G. Subramanian, D. Perez, B.P. Uberuaga, C.N. Tomé, and A.F. Voter, Method to account for arbitrary strains in kinetic Monte Carlo simulations, Phys. Rev. B 87 (2013), p. 144107.

[44] R. Gibala, W.A. Counts, and C. Wolverton, The hydrogen cold work peak in bcc iron: Revisited, with first principles calculations and implications for hydrogen embrittlement, Mater. Res. 21 (2018), p. e20170868.

[45] R. Oriani, The diffusion and trapping of hydrogen in steel, Acta Metall. 18 (1970), pp. 147-157.

[46] B. Puchala, M.L. Falk, and K. Garikipati, An energy basin finding algorithm for kinetic monte carlo acceleration, J. Chem. Phys. 132 (2010), p. 134104.

[47] A.J. Kumnick and H.H. Johnson, Deep trapping states for hydrogen in deformed iron, Acta Metall. 28 (1980), pp. 33-39.

[48] D. Tanguy, $\mathrm{H}$ effects in Al-Mg, Al-Zn-Mg alloys, and Al: Experiments, continuum, and atomistic modeling, Corrosion 72 (2016), pp. 297-313.

[49] H. Saitoh, Y. Iijima, and K. Hirano, Behaviour of hydrogen in pure aluminium, Al-4 mass\% $\mathrm{Cu}$ and Al-1 mass\% $\mathrm{Mg}_{2}$ Si alloys studied by tritium electron microautoradiography, J. Mater. Sci. 29 (1994), pp. 5739-5744. 